\title{
Dysfunctional personality traits: relationship with Five Factor Model, adaptation and symptomatology in a community sample from Buenos Aires
}

\author{
Juliana Beatriz Stover, ${ }^{1,2}$ Alejandro Castro Solano, ${ }^{1,3}$ Mercedes Fernández Liporace ${ }^{1,2}$ \\ ${ }^{1}$ National Scientific and Technical Research Council (CONICET), Buenos Aires; ${ }^{2}$ Faculty of Psychology, University of Buenos \\ Aires; ${ }^{3}$ Faculty of Social Sciences, University of Palermo, Buenos Aires, Argentina
}

\begin{abstract}
The paper introduces an analysis of the dimensional maladaptive personality traits model stated in the section III of Diagnostic and Statistical Manual of Mental Disorders, 5th Edition (DSM-5), conducted on a community sample composed of 906 adults from Buenos Aires, Argentina. Data were gathered using a socio-demographic survey as well as the Personality Inventory for DSM-5 (PID-5), the Big Five Inventory (BFI), the Symptom Check List 90-R (SCL-90-R), and the World Health Organization Disability Assessment Schedule 2.0 (WHODAS 2.0). A joint exploratory factor analysis was carried out including the PID-5's 25 facets along with BFI's 5 factors as input variables. Findings showed a 5-factor structure mostly coincident with DSM-5's hypotheses as well as with previous research. Besides, correlations calculated between PID-5 and BFI scores behaved according to theoretical hypotheses. By means of a two-stage cluster analysis which used WHODAS 2.0 score and the Global Severity Index index from SCL-90-R as segmentation criteria, two groups were differentiated: The High-Adaptation/Low-Symptomatology group vs the LowAdaptation/High-Symptomatology one. The second group obtained significantly higher means in the five domains, and in 24 of the 25 facets of PID-5.
\end{abstract}

Key words: Personality; Dysfunctional traits; Five Factor Model.

Correspondence: Juliana Beatriz Stover, Faculty of Psychology, University of Buenos Aires, Gral. Juan Lavalle 2353 (C1052AAA), Buenos Aires, Argentina.

Tel.: +54.11.4952.5481/5490

E-mail: julianastover@psi.uba.ar

Citation: Stover, J. B., Castro Solano, A., \& Fernández Liporace, M. (2019). Dysfunctional personality traits: relationship with Five Factor Model, adaptation and symptomatology in a community sample from Buenos Aires. Research in Psychotherapy: Psychopathology, Process and Outcome, 22(2), 281-291. doi: 10.4081/ripppo.2019.343

Contributions: the authors contributed equally.

Conflict of interest: the authors declare no potential conflict of interest.

Funding: this work was supported by the National Council of Scientific and Technological Investigations (CONICET) and by the University of Buenos Aires under Grant UBACyT 20020170100064BA.

Received for publication: 9 November 2018.

Revision received: 5 April 2019.

Accepted for publication: 26 April 2019.

This work is licensed under a Creative Commons Attribution NonCommercial 4.0 License (CC BY-NC 4.0).

${ }^{\circ}$ Copyright: the Author(s), 2019

Licensee PAGEPress, Italy

Research in Psychotherapy:

Psychopathology, Process and Outcome 2019; 22:281-291

doi:10.4081/ripppo.2019.343

\section{Introduction}

The third section of the Diagnostic and Statistical Manual of Mental Disorders, fifth edition (DSM-5; American Psychiatric Association, 2013a) introduces a major change in the classification of personality disorders. It consists in the incorporation of the dimensional Five Factor model which has been added to the former DSM-5's categorical approach. Such a dimensional approach hypothesizes disorders as maladaptive traits of the normal personality. This perspective is opposite to the traditional categorical taxonomy included in the section II of DSM5 (also currently in force), where disorders are defined as discrete clinical syndromes, therefore different and unique from a qualitative viewpoint.

The dimensional model, conversely, assumes the continuity between normal and pathological personality. On these grounds, 25 lower order personality facets are placed in the dysfunctional pole of that continuum - Anhedonia, Anxiousness, Attention Seeking, Callousness, Deceitfulness, Depressivity, Distractibility, Eccentricity, Emotional Lability, Grandiosity, Hostility, Impulsivity, Intimacy Avoidance, Irresponsibility, Manipulativeness, Perceptual Dysregulation, Perseveration, Restricted Affectivity, Rigid Perfectionism, Risk Taking, Separation Insecurity, Submissiveness, Suspiciousness, Unusual Beliefs, and Experiences, Withdrawal. Such low order facets are grouped into five higher order domains named Nega- 
tive Affectivity, Detachment, Antagonism, Disinhibition, and Psychoticism. These domains are conceived as the reverse of the main dimensions which compose the Five Factor Model (FFM). The original FFM model described personality in terms of the combination of Neuroticism, Extraversion, Openness, Agreeableness and Consciousness (Costa \& McCrae, 1985). These current changes regarding the notion of continuum imply that the five major domains present a gradient which runs from one pole to another-functional vs dysfunctional - throughout a whole range of possible variations.

DSM-5's task force promotes research on this model, aiming at gathering sound supporting empirical evidence (Hopwood et al., 2013). The Personality Inventory for DSM-5 (PID-5; Krueger, Derringer, Markon, Watson, \& Skodol, 2012, 2013) was developed to achieve this goal. It is a self-report designed to assess the 25 pathological facets as well as the higher order pathological domains. Its 220 items are responded by means of 4-point Likert scale: Very False or Often False, Sometimes or Somewhat False, Sometimes or Somewhat True, Very True or Often True. In addition, its 5-factor structure was verified by several studies (Al-Dajani, Gralnick, \& Bagby, 2015) in samples collected from community populations (e.g., Krueger et al., 2012), clinical populations (e.g., Quilty, Ayearst, Chmielewski, Pollock, \& Bagby, 2013), and in versions adapted to different languages as well -i.e., Italian (Fossati, Krueger, Markon, Borroni, \& Maffei, 2013), German (Zimmerman et al., 2014), Spanish (Gutiérrez et al., 2015), French (Roskam et al., 2015), Danish (Bastiaens et al., 2016), Norwegian (Thimm, Jordan, \& Bach, 2016), and Arabian (Al-Attiyah, Megreya, Alrashidi, Dominguez-Lara, \& Al-Sheerawi, 2017). All these studies reported an adequate internal consistency (e.g., Al-Dajani et al., 2015) whilst other studies found an optimal temporal stability in scores (e.g., Pires, Ferreira, \& Guedes, 2017; Pires et al., 2018; Suzuki, Griffin, \& Samuel, 2016).

PID-5 also includes an informant-report version (PID5-IRF; Markon, Quilty, Bagby, \& Krueger, 2013), intended to add information from external sources when other people's perceptions or relationships become a matter of interest in the assessment process. Moreover, two new versions of PID-5 were published: the PID-5 Short Form (PID-5SF; Maples et al., 2015) composed by 100 items, and the PID-5 Brief Form (PID-5-BF; APA, 2013c; Góngora \& Castro Solano, 2017), an even shorter 25-item form.

Several studies tested possible associations between the DSM-5's section III model and the FFM in view of the importance of the underlying hypothesis which states such linkage which is, at the same time, one of the major changes entailed in the dimensional model of maladaptive personality traits. Therefore, studies on the continuity of normal personality traits and their pathological counterparts were carried out. Hence, correlations between PID5 scores and scores from the Big Five Inventory (BFI) and the Revised NEO Personality Inventory (NEO-PI-R) were calculated. Most results have shown the same pattern: on the one hand Negative Affectivity and Neuroticism were positively correlated whereas pairs such as Detachment and Extraversion, Antagonism and Agreeableness, Disinhibition and Consciousness obtained negative coefficients. Psychoticism and Openness, on the other hand, exhibited nil indices (e.g., Al-Dajani et al., 2015; Góngora $\&$ Castro Solano, 2017; Fowler et al., 2016; Maples et al., 2015; Pires et al., 2018; Quilty et al., 2013; Zimmerman et al., 2014). A recent study conducted in Argentina using PID-5-BF form reported the same correlational pattern (Góngora \& Castro Solano, 2017).

Openness showed a different panorama since it was associated with other PID-5 domains such as Detachment, with a negative sign (Few et al., 2013). Moreover, some studies have excluded Openness from the analyses on the grounds of the results above mentioned (e.g., Al-Attiyah et al., 2017). Other authors reported non-significant or significant moderate correlations between Psychoticism and other domains (Watson, Stasik, Ro, \& Clark, 2013).

Research using exploratory factor analysis of PID-5's 25 facets and the FFM's five factors taken together stood out as well. De Fruyt et al. (2013) found an adequate fit for a 5-factor structure, where facets have grouped with its corresponding factor of normal personality. For instance, Neuroticism loaded positively with Negative Affectivity facets whereas negative values were obtained for pairs such as Extraversion and Detachment, Agreeableness and Antagonism, Consciousness and Disinhibition. Nevertheless, a different result was obtained for Openness. It loaded on the one hand, with a positive sign, along with the Psychoticism facets - Unusual Beliefs and Experiences, Perceptual Dysregulation, Eccentricity - and along with Perseveration on the other.

Thomas et al. (2013) also reported a 5-factor solution where three factors verified theoretical hypotheses, loading as expected and with the expected sign. Thus, BFI Neuroticism along with its PID-5 facets loaded positively in a factor whereas Agreeableness and Extraversion -both with negative loads - joined with their PID-5 facets in the other two remaining factors. Yet, Consciousness, behaved in an unexpected way according to theory, loading with a positive sign along with Rigid Perfectionism, and with a negative sign with Impulsivity. Openness showed unusual results as well since the three facets of Psychoticism obtained positive loads along with the Impulsivity facet, as reported in the study by De Fruyt et al. (2013). Suzuki, Samuel, Pahlen, and Krueger (2015) reached similar conclusions employing the Item Response Theory approach to analyze the International Personality Items Pool-NEO PI-R (IPIP-NEO): Openness and the Psychoticism facets did not load together. Such a finding seemed odd according to the DSM-5 theoretical hypotheses.

The deviation in the hypothesized continuity between the dimensional model of maladaptive personality traits and the FFM which arose from the former analyses - fac- 
tor analyses and correlations - regarding Psychoticism and Openness could be explained by the way of measuring Openness. According to DeYoung, Grazioplene, and Peterson (2012), the Openness factor can be divided into Openness and Intellect factors. However, tests traditionally used to measure Openness such as NEO-PI- R or BFI do not make that differentiation. As a result, the revision of how Openness must be measured has been suggested (Góngora \& Castro Solano, 2017).

The usefulness of the model to differentiate personality facets in community samples compared to clinical samples was also examined. Bach, Maples-Keller, Bo, and Simonsen (2016) found, employing a group-contrast methodology, significantly higher means in 21 facets in patients, except for Grandiosity, Attention Seeking, Risk Taking and Manipulativeness, where no significant differences were verified. Conducting the study on a new sample, Bach, Sellbom, and Simonsen (2018) reported higher means for the clinical sample, except for Grandiosity and Risk Taking, where non-patients obtained significantly higher means. Instead, the Antagonism domain and the Manipulativeness and Attention Seeking facets did not verify statistically significant differences.

As for studies conducted with scales of the PID-5 Spanish version comparing a clinical sample $v s$ a community sample, Gutiérrez et al. (2015) also reported higher means for each facet and domain in the clinical group, though no differences were found for Grandiosity. The unique local study, carried out on a community sample using the PID-5BR, identified Detachment, Disinhibition and Negative Affectivity as accurate predictors of risk factors for mental health. At the same time, Detachment and Negative Affectivity arose as useful predictors of emotional well-being (Góngora \& Castro Solano, 2017).

In sum, the literature on the research of the dimensional model of maladaptive personality traits introduced in section III of DSM-5 puts on the table a scarcity of research on Spanish speakers' populations. Argentina has no research about the matter employing PID-5 as a measurement scale. Thus, regarding the importance of examining the linkage between such model and the FFM in diverse cultures (Thomas et al., 2013), this study was aimed at: i) the analysis of the relationship between PID5 's facets and BFI factors by means of a joint factor analysis; ii) the exam of such relationship by means of correlational coefficients; and iii) the analysis of possible differences in scores when comparing groups in a community sample (Low-Symptomatology/High-Adaptation vs High-Symptomatology/Low-Adaptation). The hypotheses to be tested were the following: i) PID-5's facets would load along with their opposite BFI factor; ii) significant and positive correlations between Negative Affectivity and Neuroticism would likely to be found whereas significant and negative indices between pairs such as Detachment and Extraversion, Antagonism and Agreeableness, Disinhibition and Consciousness would be found; the correlational index between Psychoticism and Openness would be nil; iii) significantly higher means would be found for the de High-Symptomatology/LowAdaptation group in domains and facets when compared to the Low-Symptomatology/ High-Adaptation group.

\section{Methods}

\section{Participants}

The sample was composed of 906 adult non-patients from Buenos Aires and its outskirts (49.9\% males, 51.1\% females), with ages between 18 and 87 years old ( $M=40.72$; $S D=17.40$ ). Regarding their educational level, most of the examinees reported achieving higher education since $40.3 \%$ were college undergraduates, and $30.4 \%$ were college graduates. The remaining percentages were as follows: $15.6 \%$ were high school graduates, $7.2 \%$ had not finished their high school studies, $5.6 \%$ were graduates from elementary school, and $0.9 \%$ had not finished elementary school. As for marital status, similar proportions of single and married persons were reported (42.6\% vs 42.7\%). There was a lower percentage of divorced people (11\%) and widowers (3.6\%). Most of them lived with their couples and/or children (48\%). The rest lived with their parents (23.8\%), alone (16.1\%), with friends $(7.9 \%)$, and with other people $(4.1 \%)$. Finally, the self- perceived socioeconomic level was mostly medium (77.4\%). The other categories clustered as follows: medium-low (11.4\%), medium-high (9.7\%), low (1.3\%) and high (0.2\%).

\section{Materials}

\section{Socio-demographic survey}

It gathers data on gender, age, marital status, living situation, educational level and perceived socioeconomic level.

\section{Personality Inventory for DSM-5}

PID-5 (APA, 2013b; Fernández Liporace \& Castro Solano, 2015; Krueger et al., 2012, 2013) is composed of 220 items with a 4-point Likert response scale and measures the above-mentioned 25 pathological personality facet traits as well as the five major domains.

\section{Big Five Inventory}

BFI (Castro Solano \& Casullo, 2001; John, 1990) includes 44 items to be responded by a 5 -point Likert scale measuring the five big factors of personality according to FFM model (Extraversion, Agreeableness, Consciousness, Neuroticism, and Openness).

\section{Symptom Check List 90-R}

The Symptom Check List 90-R (SCL-90-R; Casullo, 2008; Derogatis, 1983) assesses psychopathologic symp- 
tomatology suffered in the last week by means of scales such as Somatization, Obsessions-Compulsions, Interpersonal Sensitivity, Depression, Anxiety, Hostility, Phobic Anxiety, Paranoid Ideation, and Psychoticism. Additionally, seven critical items and three index scores - Global Severity Index, Positive Symptoms Total Index, and Positive Symptom Distress Index- can be calculated as a general summary of scales' results. SCL-90-R consists of 90 items with a 5-point Likert response.

\section{World Health Organization Disability Assessment Schedule 2.0}

The World Health Organization Disability Assessment Schedule 2.0 (WHODAS 2.0; Üstün et al., 2010) scale assesses the degree of health and adaptation in non-patients as well as in patients in six domains - Cognition, Mobility, Self-Care, Getting Along, Life Activities, Participation - summarized in a total score. The 12-item and 5-point Likert scale version was employed in this study.

\section{Procedure}

Participants, who previously volunteered, were contacted via e-mail. They received information about the study goals and were invited to participate. Appointments were scheduled with those who responded affirmatively. Data gathering was carried throughout a whole year, in individual sessions in the different sites of the Faculty of Psychology, conducted by trained psychologists, researchers from the University of Buenos Aires. Participants signed an agreement where they were informed about the research goals, and they received the assurance on the data confidentiality and anonymity as they accepted to join in. They were informed about the possibility of declining participation or interrupting response at any moment.

About data analyses, performed using the PASW package, Version 18.0 (SPSS Inc., 2009), descriptive statistics (means, standard deviations, skewness, and kurtosis) were calculated for the five domains and the 25 facets of PID-5, as well as for the BFI factors. Internal consistency was estimated by means of the Cronbach's Alphas indices.

An exploratory factor analysis (EFA) was carried out using the default oblique Geomin rotation and maximum likelihood estimation method, taking the 25 PID-5 facets and the five BFI factors as input variables. To do so the Mplus6 software was used (Muthén \& Muthén, 19982011). Following Hayton, Allen, and Scarpello (2004), a parallel analysis was performed to establish the number of factors to be extracted (Horn, 1965). The congruence between the components reported in this study and the former by Thomas et al. (2013) and by De Fruyt et al. (2013) was examined using Orthosim (Barrett, 2005), by means of an orthogonal configural target rotation without row-normalization (non-Procrustes).

Pearson's $r$ coefficients between BFI dimensions and the domains and facets of PID-5 were calculated. Owing to the large number of correlations tested, the Benjamini and Hochberg (1995) procedure was used, due to it de- creases the false discovery rate. Finally, a two-stage clusters procedure was performed to differentiate two groups according to the severity of the general symptomatology they were suffering from, and the degree of adaptation achieved. In other words, the purpose consisted in weighing up the degree of non-specific symptomatic affectation and the level of general functioning simultaneously. Such estimation was carried out by combining the values of the SCL-90-R's Global Severity Index (Casullo, 2008; Derogatis, 1983) along with the WHODAS 2.0 total score (Üstün et al., 2010). This way, the High-Adaptation/Low-Symptomatology $(n=684)$ group and the Low-Adaptation/High-Symptomatology group $(n=216)$ were compared. These analyses were also carried out with the PASW software, Version 18.0 (SPSS Inc., 2009).

\section{Results}

\section{Descriptive statistics}

First, skewness and kurtosis were examined. Most values moved within the +/- 2 range. Regarding internal consistency, Cronbach's Alphas indices were adequate for domains (.85 to .93). As for facets, most of them obtained values over .70, with a higher index of .91 for Eccentricity. Low Alphas were found for three facets, Intimacy Avoidance (.52), Irresponsibility (.57), and Suspiciousness (.54). Values were appropriate for BFI factors, except for the case of Agreeableness (.68) (Table 1).

\section{Joint exploratory factor analysis}

Five eigenvalues of the correlation matrix $(9.19,2.91$, $2.24,1.90,1.46)$ were higher than those obtained from the parallel analysis $(1.35,1.30,1.26,1.23,1.21)$. The sixth factor showed a difference of 0.03 (1.21 vs 1.18). Owing to that, the 6-factor and 5-factor structures were tested. Despite of the fact that the parallel analysis suggested 6 factors to be retained, such a solution seemed theoretically weak. Hence, the 5 -factor one arose as the most sensible alternative.

EFA results showed that the first four BFI factors along with their PID-5 counterpart facets loaded as expected by DSM-5's hypotheses as well as by previous research. BFI Neuroticism on the one hand, as well as PID-5 Anhedonia, Anxiousness, Depressivity, Emotional Lability, Perseveration and Separation Insecurity were grouped, with positive loads higher than .40, in Factor 1. BFI Consciousness and PID-5 Rigid Perfectionism joined with negative loads in Factor 2, along with PID-5 Distractibility and Irresponsibility, which loaded positively. Factor 3 grouped BFI Agreeableness, with a negative load, and PID-5 Attention Seeking, Callousness, Deceitfulness, Grandiosity and Manipulativeness, with positive loads. Factor 4 assembled BFI Extraversion with a negative load, and PID-5 Restricted Affectivity and Withdrawal, 
both positively loaded. Factor 5 was composed of PID-5 Eccentricity, Perceptual Dysregulation, Unusual Beliefs and Experiences with positive loads. None of BFI dimensions loaded on Factor 5 with values equal to/or over 40 (Openness was the higher, with .369). Finally, PID-5 Hostility, Impulsivity, Intimacy Avoidance, Risk Taking, and Submissiveness Suspiciousness facets did not group since they obtained loads lower than .40. Table 2 shows factorial loadings over .40 , in bold.

When comparing this factor structure with the one reported by Thomas et al. (2013) a good overall solution congruence was found (.92). The same happened with Factors 1 (.97), 3 (.93), 4 (.92), and 5 (.95) since their indices were over .90 (Barrett, 2005). Nevertheless, the congruence was moderate for Factor 2, with a lower value (.89). About De Fruyt et al.'s studies (2013) values were higher than Thomas' for the overall solution congruence (.94), as well as for each factor $(\mathrm{F} 1=.97, \mathrm{~F} 2=.94, \mathrm{~F} 3=.95, \mathrm{~F} 4=.93, \mathrm{~F} 5=.90)$.

\section{Correlations between pathological personality traits and normal personality traits}

To analyze associations between BFI and PID-5 scores, cut-off points criteria established by Cohen (1988) for the effect size ( 0.30 medium, 0.50 large) were followed for evaluating Peason's coefficients. Values behaved as expected considering the opposite poles hypothesized as defining the continuity from normal to pathologic personality introduced in DSM-5 (American Psychiatric Association, 2013a), which is understood, in fact, as an antipodal linkage -normal vs pathologic-. Extraversion and Detachment (-.523) largely correlated with a negative sign, as well as Consciousness and Disinhibi-

Table 1. Personality Inventory for Diagnostic and Statistical Manual of Mental Disorders, 5th Edition. Descriptive statistics and internal consistency.

\begin{tabular}{|c|c|c|c|c|c|}
\hline & \multicolumn{5}{|c|}{ Community Sample (N=906) } \\
\hline & Mean & SD & Skewness & Kurtosis & $\alpha$ \\
\hline \multicolumn{6}{|l|}{ PID-5 Domains } \\
\hline Negative Affectivity & 22.52 & 11.08 & 0.61 & -0.04 & .87 \\
\hline Detachment & 15.65 & 10.00 & 1.00 & 0.83 & .85 \\
\hline Antagonism & 11.00 & 8.69 & 1.19 & 1.60 & .87 \\
\hline Disinhibition & 16.87 & 10.04 & 0.50 & -0.35 & .86 \\
\hline Psychoticism & 14.78 & 13.74 & 1.38 & 1.96 & .93 \\
\hline \multicolumn{6}{|l|}{ PID-5 Facets } \\
\hline Anhedonia & 5.46 & 3.89 & 0.95 & 0.88 & .74 \\
\hline Anxiousness & 10.43 & 5.21 & 0.52 & -0.18 & .78 \\
\hline Attention Seeking & 6.64 & 5.21 & 0.77 & 0.03 & .85 \\
\hline Callousness & 5.03 & 5.10 & 1.77 & 4.42 & .78 \\
\hline Deceitfulness & 4.94 & 4.13 & 1.46 & 2.62 & .77 \\
\hline Depressivity & 4.42 & 5.11 & 1.97 & 4.91 & .83 \\
\hline Distractibility & 7.43 & 5.53 & 0.61 & -0.25 & .84 \\
\hline Eccentricity & 7.01 & 7.34 & 1.36 & 1.69 & .91 \\
\hline Emotional Lability & 7.82 & 4.16 & 0.39 & -0.27 & .71 \\
\hline Grandiosity & 2.95 & 3.13 & 1.32 & 1.71 & .72 \\
\hline Hostility & 10.32 & 5.14 & 0.52 & -0.09 & .74 \\
\hline Impulsivity & 5.96 & 4.27 & 0.60 & -0.24 & .83 \\
\hline Intimacy Avoidance & 4.17 & 3.20 & 1.03 & 0.84 & .52 \\
\hline Irresponsibility & 3.47 & 2.95 & 0.90 & 0.36 & .57 \\
\hline Manipulativeness & 3.11 & 2.99 & 0.92 & 0.15 & .76 \\
\hline Perceptual Dysregulation & 4.60 & 4.64 & 1.45 & 2.15 & .78 \\
\hline Perseveration & 7.28 & 4.94 & 0.64 & -0.01 & .78 \\
\hline Restricted Affectivity & 6.40 & 3.99 & 0.53 & -0.36 & .70 \\
\hline Rigid Perfectionism & 10.34 & 6.53 & 0.59 & -0.20 & .85 \\
\hline Risk Takings & 16.15 & 7.11 & 0.18 & -0.09 & .81 \\
\hline Separation Insecurity & 4.27 & 4.24 & 1.18 & 0.95 & .81 \\
\hline Submissiveness & 3.12 & 2.38 & 0.64 & -0.09 & .71 \\
\hline Suspiciousness & 6.58 & 3.36 & 0.61 & 0.44 & .54 \\
\hline Unusual Beliefs and Experiences & 3.17 & 3.90 & 1.67 & 2.86 & .75 \\
\hline Withdrawal & 6.02 & 5.70 & 1.23 & 1.33 & .86 \\
\hline \multicolumn{6}{|l|}{ BFI Factors } \\
\hline Extraversion & 28.02 & 5.66 & -0.14 & -0.47 & .74 \\
\hline Agreeableness & 35.48 & 5.25 & -0.38 & -0.14 & .68 \\
\hline Consciousness & 34.17 & 6.20 & -0.33 & -0.42 & .77 \\
\hline Neuroticism & 22.00 & 5.81 & 0.11 & -0.43 & .73 \\
\hline Openness & 36.81 & 6.55 & -0.18 & -0.36 & .75 \\
\hline
\end{tabular}

SD, standard deviation; PID-5, Personality Inventory for Diagnostic and Statistical Manual of Mental Disorders, 5th Edition; BFI, Big Five Inventory. 
tion (-.624). Neuroticism and Negative Affectivity were positively and largely associated (.587). Agreeableness and Antagonism (-.363) obtained a medium and negative coefficient whereas Openness and Psychoticism correlated in a non-significant way.

Regarding the associations between PID-5 facets scores and BFI scores, only those indices with large coefficients will be considered (Cohen, 1988). On the one hand, negative correlations were found between Extraversion and Withdrawal (-.563). The same happened between Agreeableness and Callousness (-.532), between Con- sciousness and Distractibility (-.618), and Consciousness and Irresponsibility (-.582). On the other hand, Neuroticism was positively associated with Anxiousness (.566). Finally, Openness did not obtain significant correlation indices with any facet (Table 3).

\section{Group differences}

Student's $t$ tests were calculated to analyze differences in the five domains, splitting the sample into two groups: the High-Adaptation/Low-Symptomatology one $v s$ the

Table 2. Personality Inventory for Diagnostic and Statistical Manual of Mental Disorders, $5^{\text {th }}$ Edition and Big Five Inventory joint exploratory factor analysis. Factor loadings.

\begin{tabular}{|c|c|c|c|c|c|}
\hline & $\mathbf{F 1}$ & F2 & F3 & F4 & F5 \\
\hline Extraversion & -.020 & -.126 & .139 & -.689 & .021 \\
\hline Agreeableness & -.012 & -.181 & -.511 & -.359 & .272 \\
\hline Consciousness & -.012 & -.852 & -.081 & -.047 & .003 \\
\hline Neuroticism & .756 & .106 & -.022 & .008 & -.216 \\
\hline Openness & -.143 & -.086 & .007 & -.380 & .369 \\
\hline Anxiousness & .874 & -.111 & -.024 & -.050 & -.028 \\
\hline Separation Insecurity & .668 & .003 & .044 & -.151 & -.034 \\
\hline Emotional Lability & .662 & .000 & -.083 & -.213 & .249 \\
\hline Perseveration & .545 & .108 & .042 & .063 & .305 \\
\hline Depressivity & .538 & .192 & .042 & .171 & .090 \\
\hline Anhedonia & .427 & .176 & -.005 & .376 & -.035 \\
\hline Irresponsibility & .021 & .551 & .308 & .025 & .109 \\
\hline Distractibility & .308 & .541 & -.014 & .064 & .231 \\
\hline Rigid Perfectionism & .504 & -.479 & .114 & .101 & .127 \\
\hline Deceitfulness & .030 & .055 & .853 & -.009 & -.093 \\
\hline Manipulativeness & .036 & -.114 & .838 & -.048 & -.036 \\
\hline Attention Seeking & .197 & .002 & .650 & -.339 & .050 \\
\hline Callousness & -.078 & .012 & .617 & .370 & .061 \\
\hline Grandiosity & -.013 & -.166 & .587 & .038 & .164 \\
\hline Withdrawal & .092 & -.110 & .015 & .704 & .224 \\
\hline Restricted Affectivity & -.011 & .023 & .218 & .557 & .243 \\
\hline Perceptual Dysregulation & .277 & .191 & .005 & .008 & .640 \\
\hline Unusual Beliefs and Experiences & .160 & -.065 & .029 & -.032 & .656 \\
\hline Eccentricity & .157 & .136 & .121 & .085 & .570 \\
\hline Hostility & .383 & -.105 & .387 & .205 & .014 \\
\hline Impulsivity & .339 & .201 & .155 & -.209 & .173 \\
\hline Intimacy Avoidance & .114 & -.020 & -.095 & .326 & .192 \\
\hline Risk Taking & -.308 & .177 & .334 & -.137 & .293 \\
\hline Submissiveness & .377 & .084 & .121 & -.049 & -.079 \\
\hline Suspiciousness & .341 & -.088 & .219 & .201 & .101 \\
\hline
\end{tabular}

Bold indicates loadings greater than .40 . 
Low-Adaptation/High-Symptomatology group. Cohen's $d$ statistic (Cohen, 1988) was used to examine effect sizes. This author's cut-off points criteria were followed: 0.20 (small), 0.50 (medium), 0.80 (large). The Low-Adaptation/High-Symptomatology group showed higher means in every domain. The effect size was large for Negative Affectivity, Psychoticism, Detachment and Disinhibition, and small for Antagonism (Table 4).

The 25 PDI-5 trait facets were also compared. Every facet, except for Risk Taking verified significant differences. Higher means were found for the Low Adapta-
tion/High-Symptomatology group and higher effect sizes were verified for Perserveration $(d=1.04)$, Perceptual Dysregulation $(d=0.99)$, Anxiousness $(d=0.97)$, Depressivity ( $d=0.96)$, Anhedonia $(d=0.85)$, Eccentricity $(d=0.85)$, and Distractibility $(d=0.85)$ (Table 5$)$.

\section{Discussion}

The study was aimed at examining the maladaptive personality traits model introduced in section III of

Table 3. Personality Inventory for Diagnostic and Statistical Manual of Mental Disorders, $5^{\text {th }}$ Edition and Big Five Inventory. Association indices.

\begin{tabular}{|c|c|c|c|c|c|}
\hline & Extraversion & Agreeableness & Consciousness & Neuroticism & Openness \\
\hline Negative affectivity & -.120 & -.125 & -.176 & $.587 * *$ & -.044 \\
\hline Detachment & $-.523 *$ & -.274 & -.231 & $.347^{*}$ & -.258 \\
\hline Antagonism & .026 & $-.363^{*}$ & -.169 & .111 & .068 \\
\hline Disinhibition & -.120 & -.289 & $-.624 * *$ & $.396^{*}$ & -.085 \\
\hline Psychoticism & -.136 & -.208 & -.273 & .290 & .141 \\
\hline Anhedonia & $-.376^{*}$ & -.284 & $-.331 *$ & $.435^{*}$ & -.258 \\
\hline Anxiousness & -.174 & -.158 & -.124 & $.566 * *$ & -.078 \\
\hline Attention Seeking & .199 & -.177 & -.145 & .140 & .206 \\
\hline Callousness & -.168 & $-.532 *$ & -.205 & .172 & -.105 \\
\hline Deceitfulness & -.019 & $-.386^{*}$ & -.241 & .132 & .002 \\
\hline Depressivity & -.275 & -.265 & $-.361 *$ & $.481 *$ & -.107 \\
\hline Distractibility & -.228 & -.219 & $-.618 * *$ & $.339 *$ & -.228 \\
\hline Eccentricity & -.149 & -.240 & -.279 & .270 & -.149 \\
\hline Emotional Lability & -.029 & -.043 & -.144 & $.489 *$ & .082 \\
\hline Grandiosity & .044 & -.213 & -.043 & .039 & .106 \\
\hline Hostility & -.188 & $-.449 *$ & -.136 & $.442 *$ & -.078 \\
\hline Impulsivity & .088 & -.168 & -.264 & $.328^{*}$ & -.018 \\
\hline Intimacy Avoidance & -.174 & -.084 & -.100 & .125 & -.172 \\
\hline Irresponsibility & -.109 & $-.332 *$ & $-.582 * *$ & .239 & -.060 \\
\hline Manipulativeness & .055 & -.299 & -.114 & .101 & .084 \\
\hline Perceptual Dysregulation & -.146 & -.192 & $-.306^{*}$ & $.318^{*}$ & .048 \\
\hline Perseveration & -.213 & -.210 & $-.286^{*}$ & $.432 *$ & -.099 \\
\hline Restricted Affectivity & $-.385^{*}$ & $-.360 *$ & -.221 & .181 & -.162 \\
\hline Rigid Perfectionism & -.090 & -.109 & .218 & .255 & -.003 \\
\hline Risk Takings & .133 & -.156 & -.140 & -.076 & .221 \\
\hline Separation Insecurity & -.072 & -.088 & -.165 & $.359^{*}$ & -.099 \\
\hline Submissiveness & -.105 & -.090 & -.176 & .212 & -.124 \\
\hline Suspiciousness & -.161 & -.266 & -.116 & $.317^{*}$ & -.092 \\
\hline Unusual Beliefs and Experiences & -.026 & -.053 & -.072 & .137 & .153 \\
\hline Withdrawal & $-.563 * *$ & -.240 & -.123 & .242 & -.180 \\
\hline
\end{tabular}

Bold indicates moderate and large correlations. ${ }^{*} * \mathrm{P}<.01 ; * \mathrm{P}<.05$. 
DSM-5 (American Psychiatric Association, 2013a), using the PID-5 in a community sample from Buenos Aires, Argentina. Prior to the analyses, descriptive statistics and the internal consistency of the variables to be analyzed were calculated. The data distribution was symmetric and mesokurtic for the five domains and the 25 facets of PID-5, as well as for the BFI five factors. The internal consistency analyzed for PID-5's scores

Table 4. Personality Inventory for Diagnostic and Statistical Manual of Mental Disorders, $5^{\text {th }}$ Edition domains by adaptation and symptomatology. Student's t tests.

\begin{tabular}{lcccc}
\hline & High-Adaptation/Low-Symptoms & Low-Adaptation/High-Symptoms & $\boldsymbol{t}$ & $\boldsymbol{d}$ \\
\hline Negative Affectivity & $19.91(9.69)$ & $30.76(11.16)$ & $-13.81^{* *}$ & 1.07 \\
\hline Detachment & $13.72(9.01)$ & $21.81(10.56)$ & $-11.02^{* *}$ & 0.86 \\
\hline Antagonism & $10.27(8.47)$ & $13.38(9.04)$ & $-4.62^{* *}$ & 0.36 \\
\hline Disinhibition & $14.90(9.15)$ & $23.17(10.21)$ & $-11.24^{* *}$ & 0.87 \\
\hline Psychoticism & $11.79(11.45)$ & $24.38(15.98)$ & $-12.71^{* *}$ & 0.99 \\
\hline
\end{tabular}

M, Mean; SD, standard deviation; $t$, Student's $t$ test $;$, Cohen's $d ; * * \mathrm{P}<.01$.

Table 5. Personality Inventory for Diagnostic and Statistical Manual of Mental Disorders, $5^{\text {th }}$ Edition facets by adaptation and symptomatology. Student's t tests.

\begin{tabular}{|c|c|c|c|c|}
\hline & & & $t$ & $d$ \\
\hline High-A & aptation/Low-Symptoms & Low Adaptation/High-Symptoms & & \\
\hline Anhedonia & $4.68(3.45)$ & $7.98(4.17)$ & $-11.65^{* *}$ & 0.85 \\
\hline Anxiousness & $9.29(4.65)$ & $13.95(5.27)$ & $-12.42 * *$ & 0.97 \\
\hline Attention Seeking & $6.19(5.12)$ & $8.11(5.30)$ & $-4.77 * *$ & 0.37 \\
\hline Callousness & $4.49(4.91)$ & $6.76(5.33)$ & $-5.78^{* *}$ & 0.45 \\
\hline Deceitfulness & $4.63(3.91)$ & $5.97(4.64)$ & $-4.20 * *$ & 0.32 \\
\hline Depressivity & $3.33(3.96)$ & $7.89(6.65)$ & $-12.30 * *$ & 0.96 \\
\hline Distractibility & $6.39(5.09)$ & $10.75(5.60)$ & $-10.71 * *$ & 0.83 \\
\hline Eccentricity & $5.61(6.35)$ & $11.52(8.44)$ & $-10.95^{* *}$ & 0.85 \\
\hline Emotional Lability & $7.02(3.88)$ & $10.35(4.01)$ & $-10.88^{* *}$ & 0.85 \\
\hline Grandiosity & $2.74(3.12)$ & $3.63(3.09)$ & $-3.69^{* *}$ & 0.28 \\
\hline Hostility & $9.57(4.90)$ & $12.70(5.12)$ & $-8.11 * *$ & 0.63 \\
\hline Impulsivity & $5.42(3.96)$ & $7.73(4.74)$ & $-7.11 * *$ & 0.55 \\
\hline Intimacy Avoidance & $3.80(2.95)$ & $5.33(3.66)$ & $-6.23 * *$ & 0.48 \\
\hline Irresponsibility & $3.09(2.71)$ & $4.69(3.35)$ & $-7.11 * *$ & 0.55 \\
\hline Manipulativeness & $2.91(2.91)$ & $3.77(3.16)$ & $-3.73^{* *}$ & 0.29 \\
\hline Perceptual Dysregulation & $3.58(3.77)$ & $7.85(5.61)$ & $-12.78 * *$ & 0.99 \\
\hline Perseveration & $6.17(4.34)$ & $10.89(5)$ & $-13.41 * *$ & 1.04 \\
\hline Restricted Affectivity & $5.92(3.85)$ & $7.86(4.05)$ & $-6.34 * *$ & 0.49 \\
\hline Rigid Perfectionism & $9.61(6.25)$ & $12.70(6.86)$ & $-6.19 * *$ & 0.48 \\
\hline Risk Taking & $16.15(6.93)$ & $16.17(7.62)$ & -0.02 & 0 \\
\hline Separation Insecurity & $3.59(3.77)$ & $6.46(4.86)$ & $-9.05^{* *}$ & 0.70 \\
\hline Submissiveness & $2.83(2.30)$ & $4.03(2.39)$ & $-6.61 * *$ & 0.51 \\
\hline Suspiciousness & $5.97(2.96)$ & $8.51(3.84)$ & $-10.20^{* *}$ & 0.79 \\
\hline Unusual Beliefs and Experiences & $2.60(3.45)$ & $5(4.64)$ & $-8.19^{* *}$ & 0.63 \\
\hline Withdrawal & $5.24(5.35)$ & $8.50(6.10)$ & $-7.53 * *$ & 0.58 \\
\hline
\end{tabular}

M, Mean; SD, standard deviation; $t$, Student's t test; $d$, Cohen's $d ; * * \mathrm{P}<.01$. 
was mostly adequate, as in previous research (e.g., AlDajani et al., 2015).

When performing the joint analysis of PID-5 and BFI structures, results were consistent with those reported by De Fruyt et al. (2013), and in a lower degree, with Thomas et al. (2013)'s findings. A 5-factor structure which coincided in a high degree with the domains introduced in DSM-5 (American Psychiatric Association, 2013a) was found. This similarity refers to the way facets were grouped with their opposite factors of normal personality in FFM (Costa \& McCrae, 1985). This way, the hypothesis which stated that PID-5's facets would group along with their counterparts BFI factors was verified. The first corresponds mainly to the Negative Affectivity domain, including Anxiousness, Emotional Lability, Perseveration, Separation Insecurity and Depressivity facets, as well as BFI Neuroticism (positively loaded). The second factor joined facets belonging to the Disinhibition domain (Rigid Perfectionism, Distractibility, Irresponsibility), along with BFI Consciousness. The latter was negatively loaded as expected since it is the opposite of the Disinhibition domain. PID Attention Seeking, Callousness, Deceitfulness, Grandiosity and Manipulativeness facets loaded positively on the third factor whereas BFI Agreeableness exhibited a negative load. Because of that, Factor 3 coincides with the Antagonism domain. Fourth Factor corresponded to Detachment since it grouped PID-5 Restricted Affectivity and Withdrawal (both positive), and BFI Extraversion, with a negative load. The fifth and last factor wholly matched with the grouping hypothesized for Psychoticism in the DSM-5 (American Psychiatric Association, 2013a), joining Eccentricity, Perceptual Dysregulation as well as Unusual Beliefs and Experiences. However, this factor did not include any BFI dimension with loads over .40. The highest value was achieved by Openness (.369), which exhibited a positive load, as happened in studies conducted by De Fruyt et al. (2013) and Thomas et al. (2013).

The lack of loads over 40 in this study along with the positive sign reported in previous research put on the table a repeated deviation of results from the theoretical hypothesis on the continuity of the five domains stated in DSM-5 (American Psychiatric Association, 2013a), and FFM (Costa \& McCrae, 1985). Suzuki et al. (2015) described similar findings, with Psychoticism separated from Openness, using a different methodology such as Item Response Theory.

When analyzing the relationship between the dimensional model of maladaptive personality traits and the FFM by means of correlations, the same pattern of associations reported by several authors was reported (e.g., Al-Dajani et al., 2015; Fowler et al., 2016; Góngora \& Castro Solano, 2017; Maples et al., 2015; Pires et al., 2018; Quilty et al., 2013; Zimmerman et al., 2014). Significant correlations between PID-5 domains and facets with their FFM counterparts assessed by BFI were found, mostly verifying hypothesis \#2. About the domains, asso- ciations between Negative Affectivity and Neuroticism were positive whereas they were negative for pairs such as Detachment and Extraversion, Disinhibition and Consciousness, Antagonism and Agreeableness. The Psychoticism and Openness pair did not obtain a significant correlation. Psychoticism was not significantly associated with any BFI factor, whilst Openness did not show significant indices with the rest of PID-5 domains and facets either. As above mentioned, a likely explanation for these results could be attributable to methodological differences in the measurements of Openness (DeYoung et al., 2012), which must be reviewed in further research.

Regarding group differences (Low-Adaptation/HighSymptomatology vs High-Adaptation/Low-Symptomatology), significant differences were verified with higher means for the first group in every domain and facet (except for Risk Taking). This is coincident with hypothesis \#3 in this study. Regarding the domains, highest differences were found for Negative Affectivity. It is worthy to mention that Negative Affectivity was one of the predictors of psychological well-being with higher importance in local studies which used PID-BR (Góngora \& Castro Solano, 2017). These findings illustrate the usefulness of PID-5 to distinguish groups with different symptomatic levels in terms of severity, even in community samples. Such feature acquires importance when it comes to nonclinical psychological assessment activities where the detection of symptomatology or maladaptive functioning is required for any possible purpose.

About of the lack of differences in Risk Taking, they were alike reports made by Bach et al. $(2016,2018)$ when comparing community samples to clinical samples. That can be understood plainly as a lack of differences. However, the fact that this facet is composed of a higher number of inverse items (6 of 16 of PID-5) should not be neglected. Such an imbalance of direct and inverse items in a dimension has been pointed out as problematic by Ashton, de Vries, and Lee (2016), and the issue must be examined furtherly.

About the weaknesses of this study, the analyses performed on a community sample arise as the first and most important one since PID-5 is a scale which was developed to assess pathology. Aiming at ameliorating the unavailability of a clinical sample, two groups of participants were differentiated within the community sample. It was split according to the severity of the psychopathologic symptomatology (measured by SCL-90R's Global Severity Index) along with the general adaptation level (measured by WHODAS scale) by means of a cluster analysis procedure. Results were consistent with DSM-5's hypotheses and previous research. Anyway, in despite of these improvements introduced to lessen the lack of a clinical sample, it is imperative to replicate analyses in local patients to compare their results to non-patients'. That would be major since there is no precedent of that kind. 
The second weakness arises from the scales employed, which are all self-reports. Such a feature should be always regarded since phenomena as deliberate distortions, defensiveness, acquiescence, non-acquiescence, inattention or a lack of comprehension might be likely interferences for the proper interpretation of scores. Moreover, those response styles must be assessed even more in depth in PID-5, when item balance is uneven (inverse and direct number of items), as recommended by Ashton et al. (2016). Nevertheless, since the main goal was aimed at going on with previous studies (De Fruyt et al., 2013; Thomas et al., 2013), the balance of direct and inverse items was not altered to avoid any methodological deviation from preceding research.

\section{Conclusions}

Despite the limitations above described, the major contribution rests on being this the first study carried out in Argentina which analyzes the maladaptive personality traits model (American Psychiatric Association, 2013a) by means of the PID-5 (Fernández Liporace \& Castro Solano, 2015) in a local community sample. That becomes particularly important taking into consideration that the unique local precedent corresponds to a study on PID-BR (Góngora \& Castro Solano, 2017) whereas only one research conducted in Spain was referred to the analysis of the Spanish version of PID-5 (Gutiérrez et al., 2015). Further research will go on analyzing PID-5's usefulness to represent the maladaptive traits of personality as the reverse of the FFM.

\section{References}

Al-Attiyah, A. A., Megreya, A. M., Alrashidi, M., DominguezLara, S. A., \& Al-Sheerawi, A. (2017). The psychometric properties of an Arabic version of the Personality Inventory for DSM-5 (PID-5) across three Arabic speaking Middle Eastern countries. International Journal of Culture and Mental Health, 10(2), 197-205. doi: 10.1080/17542863. 2017.1290125

Al-Dajani, N., Gralnick, T.M., \& Bagby, R.M. (2015). A psychometric review of the Personality Inventory for DSM-5 (PID-5): Current status and future directions. Journal of Personality Assessment, 98(1), 62-81. doi: 10.1080/00223891. 2015.1107572

American Psychiatric Association (2013a). Diagnostic and Statistical Manual of Mental Disorders, 5th Ed., DSM-5. Washington DC: American Psychiatric Association.

American Psychiatric Association (2013b). The Personality Inventory DSM-5 (PID-5) Self-Report Form (full version). Retrieved from https:/www.psychiatry.org/psychiatrists/ practice/dsm/educational-resources/assessment-measures\# Personality

American Psychiatric Association (2013c). The Personality Inventory for DSM-5-Brief Form (PID-5-BF)-Adult. Retrieved from http://www.psychiatry.org/practice/dsm/dsm5/onlineassessment-measures\#Personality
Ashton, M. C., de Vries, R. E., \& Lee, K. (2016). Trait variance and response style variance in the scales of the Personality Inventory for DSM-5 (PID-5). Journal of Personality Assessment, 99(2), 192-203. doi: 10.1080/00223891.2016. 1208210

Bach, B., Maples-Keller, J. L., Bo, S., \& Simonsen, E. (2016). The alternative DSM-5 personality disorder traits criterion: A comparative examination of three self-report forms in a Danish population. Personality Disorders: Theory, Research, and Treatment, 7(2), 124-135. doi: 10.1037/ per0000162

Bach, B., Sellbom, M., \& Simonsen, E. (2018). Personality Inventory for DSM-5 (PID-5) in clinical versus nonclinical individuals: Generalizability of psychometric features. Assessment, 25(7), 815-825. doi: 10.1177/ 1073191117709070

Barrett, P. T. (2005). Orthosim 2 (version 2.0): Target-comparasion matrix fitting [Computer software and manual]. Retrieved from http://www.pbarrett.net/orthosim/orthosim.html

Bastiaens, T., Claes, L., Smits, D., De Clercq, B., De Fruyt, F., Rossi, G., ... De Hert, M. (2016). The construct validity of the Dutch Personality Inventory for DSM-5 Personality Disorders (PID-5) in a clinical sample. Assessment, 23(1), 4251. doi: $10.1177 / 1073191115575069$

Benjamini, Y., \& Hochberg, Y. (1995). Controlling the false discovery rate: A practical and powrful approach to multiple testing. Journal of the Royal Statistical Society. Series B (Methodological), 57(1), 289-300.

Castro Solano, A., \& Casullo, M. M. (2001). Facetas de personalidad, bienestar psicológico y rendimiento académico en adolescentes [Personality traits, psychological well-being and academic achievement in Argentine adolescents]. Interdisciplinaria, 18(1), 65-85.

Casullo, M. M. (2008). El Listado de Síntomas SCL-90-R de Derogatis. [The Derogatis' Symptom Checklist-90-R]. Buenos Aires: Departamento de Publicaciones, Facultad de Psicología, UBA.

Cohen, J. (1988). Statistical Power Analysis for the Behavioral Sciences. Second Edition. Hillsdale, NJ: LEA.

Costa, P. T., \& McCrae, R. R. (1985). The NEO Personality Inventory Manual. Odessa Florida: Psychological Assessment Resources.

De Fruyt, F., De Clercq, B., De Bolle, M., Wille, B., Markon, K., \& Krueger, R. F. (2013). General and maladaptive traits in a five-factor framework for DSM-5 in a university student sample. Assessment, 20(3), 295-307. doi: 10.1177/ 1073191113475808

Derogatis, L. R. (1983). Symptom Checklist-90-Revised. San Antonio: Pearson.

DeYoung, C. G., Grazioplene, R. G., \& Peterson, J. B. (2012). From madness to genius: The Openness/Intellect trait domain as a paradoxical simplex. Journal of Research in Personality, 46, 63-78. doi: 10.1016/j.jrp.2011.12.003

Fernández Liporace, M. L., \& Castro Solano, A. (2015). Personality Inventory for DSM5. Adult Form. Argentinean Version. Unpublished manuscript.

Few, L. R., Miller, J. D., Rothbaum, A. O., Meller, S., Maples, J., Terry, D. P., ... MacKillop, J. (2013). Examination of the section III DSM-5 diagnostic system for personality disorders in an outpatient clinical sample. Journal of $A b$ normal Psychology, 122(4), 1057-1069. doi: 10.1037/ a0034878 
Fossati, A., Krueger, R. F., Markon, K. E., Borroni, S., \& Maffei, C. (2013). Reliability and validity of the Personality Inventory for DSM-5 (PID-5): Predicting DSM-IV Personality disorders and psychopathy in community-dwelling Italian adults. Assessment, 20(6), 689-708. doi: 10.1177/ 1073191113504984

Fowler, J. C., Patriquin, M. A., Madan, A., Allen, J. G., Frueh, B. C., \& Oldham, J. M. (2015). Incremental validity of the PID-5 in relation to the Five Factor Model and traditional polythetic personality criteria of the DSM-5. International Journal of Methods in Psychiatric Research, 26(2), 1-9. doi: $10.1002 / \mathrm{mpr} .1526$.

Góngora, V. C., \& Castro Solano, A. (2017). Pathological personality traits (DSM-5), risk factors, and mental health. SAGE Open, 1-10. doi: 10.1177/2158244017725129

Gutiérrez, F., Aluja, A., Peri, J. M., Calvo, N., Ferrer, M., Baillés, E., ... Krueger, R. F. (2015). Psychometric properties of the Spanish PID-5 in a clinical and a community sample. Assessment, 24(3), 326-336. doi: 10.1177/1073191115606518

Hayton, J. C., Allen, D. G., \& Scarpello, V. (2004). Factor retention decisions in exploratory factor analysis: A tutorial on parallel analysis. Organizational Research Methods, 7(2), 191-205. doi: 10.1177/1094428104263675

Hopwood, C. J., Wright, A. G. C., Krueger, R. F., Schade, N., Markon, K. E., \& Morey, L. C. (2013). DSM-5 pathological personality traits and the Personality Assessment Inventory. Assessment, 20(3), 269-285. doi: 10.1177/1073191113486286

Horn, J. L. (1965). A rationale and test for the number of factors in factor analysis. Psychometrika, 30(2), 179-185. doi: 10.1007/BF02289447

John, O. (1990). The five factor taxonomy: Dimension of personality in the natural language and in questionnaires. In L. Pervin (Ed.), Handbook of Personality: Theory and Research. New York: Guilford Press.

Krueger, R. F., Derringer, J., Markon, K. E., Watson, D., \& Skodol, A. E. (2012). Initial construction of a maladaptive personality trait model and inventory for DSM-5. Psychological Medicine, 42(9), 1879-1890. doi: 10.1017/ S0033291711002674

Krueger, R. F., Derringer, J., Markon, K. E., Watson, D., \& Skodol, A. E. (2013). The Personality Inventory for DSM-5 (PID-5)-Adult. Massachusetts: American Psychiatric Association.

Maples, J. L., Carter, N. T., Few, L. R., Crego, C., Gore, W. L., Samuel, D. B., ... Miller, J. D. (2015). Testing whether the DSM-5 personality disorder trait model can be measured with a reduced set of items: An item response theory investigation of the Personality Inventory for DSM-5. Psychological Assessment, 27(4), 1195-1210. doi: 10.1037/ pas0000120

Markon, K. E., Quilty, L. C., Bagby, R. M., \& Krueger, R. F. (2013). The development and psychometric properties of an informant-report form of the personality inventory for DSM5 (PID-5). Assessment, 20(3), 370-383. doi: 10.1177/ 1073191113486513

Muthén, L. K., \& Muthén, B. O. (1998-2011). Mplus user's guide (6th ed.). Los Angeles, CA: Author.

Pires, R., Ferreira, A. S., \& Guedes, D. (2017). The psychometric properties of the Portuguese version of the Personality
Inventory for DSM-5. Scandinavian Journal of Psychology, 58, 468-475 doi: 10.1111/sjop. 12383

Pires, R., Sousa Ferreira, A., Guedes, D., Gonçalves, B., \& Henriques-Calado, J. (2018). Estudo das propriedades psicométricas - formas longa, reduzida e breve - da versão portuguesa do Inventário da Personalidade para o DSM-5 (PID-5). [Study of psychometric properties - long, short and brief forms - of the Portuguese version of the Personality Inventory for DSM-5 (PID-5)]. Revista Iberoamericana de Diagnóstico y Evaluación Psicológica, 47(2), 197-212. doi: 10.21865/RIDEP47.2.14

Quilty, L. C., Ayearst, L., Chmielewski, M., Pollock, B. G., \& Bagby, R. M. (2013). The psychometric properties of the Personality Inventory for DSM-5 in an APA DSM-5 field trial sample. Assessment, 20(3), 362-369. doi: 10.1177/ 1073191113486183

Roskam, I., Galdiolo, S., Hansenne, M., Massoudi, K., Rossier, J., Gicquel, L., \& Rolland, J.-P. (2015). The psychometric properties of the French version of the Personality Inventory for DSM-5. PLoS One, 10, e0133413. doi: 10.1371/ journal.pone. 0133413

SPSS Inc. (2009). PASW Statistics for Windows, Version 18.0. Chicago: SPSS Inc.

Suzuki, T., Griffin, S. A., \& Samuel. D. B. (2016). Capturing the DSM-5 alternative personality disorder model traits in the Five-Factor Model's Nomological Net. Journal of Personality, 124(2), 343-354. doi: 10.1111/jopy.12235

Suzuki, T., Samuel, D. B., Pahlen, S., \& Krueger, R. F. (2015). DSM-5 alternative personality disorder model traits as maladaptive extreme variants of the Five-Factor Model: An item-response theory analysis. Journal of Abnormal Psychology, 124(2), 343-354. doi: 10.1037/abn0000035

Thimm, J. C., Jordan, S., \& Bach, B. (2016). Hierarchical structure and cross-cultural measurement invariance of the Norwegian version of the Personality Inventory for DSM-5. Journal of Personality Assessment, 99(2), 204-210. doi: 10.1080/00223891.2016.1223682

Thomas, K. M., Yalch, M. M., Krueger, R. F., Wright, A. G. C., Markon, K. E., \& Hopwood, C. J. (2013). The convergent structure of DSM-5 personality trait facets and Five-Factor Model trait domains. Assessment, 20(3), 308-311. doi: $10.1177 / 1073191112457589$

Üstün, T. B., Chatterji, S., Kostanjsek, N., Rehm, J., Kennedy, C., Epping-Jordan, J., ... Pull, C. (2010). Developing the World Health Organization Disability Assessment Schedule 2.0. Bulletin of the World Health Organization, 88, 815-823. doi: 10.2471/BLT.09.067231

Watson, D., Stasik, S. M., Ro, E., \& Clark, L. A. (2013). Integrating normal and pathological personality: Relating the DSM-5 trait-dimensional model to general traits of personality. Assessment, 20(3), 312-326. doi: 10.1177/ 1073191113485810

Zimmerman, J., Altenstein, D., Krieger, T., Holtforth, M. G., Pertsch, J., Alexopoulos, J., ... Leising, D. (2014). The structure and correlates of self-reported DSM-5 maladaptive personality traits: Findings from two Germans peaking samples. Journal of Personality Disorders, 28(4), 518-540. doi: 10.1521/pedi_2014_28_130 